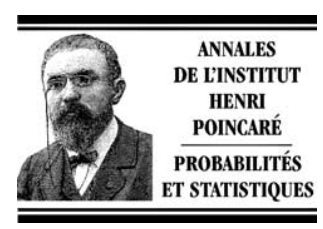

www.elsevier.com/locate/anihpb

\title{
Martin boundary theory of some quantum random walks
}

\author{
Benoît Collins \\ DMA, École normale supérieure, 45, rue d'Ulm, 75230 Paris cedex 05, France \\ Received 2 December 2002; received in revised form 18 August 2003; accepted 13 October 2003 \\ Available online 10 February 2004
}

\begin{abstract}
In this paper we define a general setting for Martin boundary theory associated to quantum random walks, and prove a representation theorem. We show that in the dual of a simply connected Lie subgroup of $U(n)$, the extremal Martin boundary is homeomorphic to a sphere. Then, we investigate restriction of quantum random walks to Abelian subalgebras of group algebras, and establish a Ney-Spitzer theorem for an elementary random walk on the fusion algebra of $S U(n)$, generalizing a previous result of Biane. We also consider the restriction of a quantum random walk on $S U_{q}(n)$ introduced by Izumi to two natural Abelian subalgebras, and relate the underlying Markov chains by classical probabilistic processes. This result generalizes a result of Biane.
\end{abstract}

(C) 2004 Elsevier SAS. All rights reserved.

\section{Résumé}

Dans cet article, nous définissons un cadre général pour la théorie de Martin associée à une large classe de marches au hasard sur le dual de groupes compacts, et établissons un théorème de représentation intégrale. Ensuite, nous montrons que dans le dual d'un sous-groupe de Lie simplement connexe de $U(n)$, la frontière de Martin extremale est homéomorphe à une sphère. Nous nous concentrons alors sur la restriction de marches au hasard quantiques à certaines sous-algèbres Abéliennes d'algèbres de groupes, et établissons un théorème de Ney-Spitzer pour une marche au hasard "de Bernoulli” sur l'algèbre de fusion de $S U(n)$. Nous considérons aussi la restriction d'une marche au hasard quantique introduite par Izumi à deux sous-algèbres abéliennes distinctes, et relions les chaînes de Markov sous-jacentes par des procédés probabilistes classiques. Ce résultat généralise un résultat de Biane.

(C) 2004 Elsevier SAS. All rights reserved.

MSC: $31 \mathrm{C} 35 ; 46 \mathrm{~L} 53$

Keywords: Martin boundary; Quantum probability theory

\section{Introduction}

The classical Martin boundary theory gives a geometric and probabilistic solution to the problem of describing positive harmonic functions with respect to a transient (sub)Markov operator. This theory is well established in the framework of random walks on classical structures.

E-mail address: benoit.collins@ens.fr (B. Collins). 
On the other hand, quantum probability became a self contained area in the 80's, short after Connes developped his non commutative geometry theory, and in the same spirit. For references, see for example [5,21-26]. Quantum probability quickly developped many independent and very active ramification such as quantum stochastic processes [1], free probability [31], and of course random matrix theory.

The problem of non-commutative harmonic analysis has also been developped for operator algebraic and group theoretic purposes, but its developments for its own probabilist sake are only at its first babblings. In particular, the study of quantum Martin boundary was initiated by the series of papers of Biane [4-9] of the early nineties, but many interesting questions raised by his papers have been left unanswered since then. The purpose of this paper is to answer some of these questions, define a reasonably general framework for quantum Martin boundary in which an integral representation theorem works (Theorem 3.1), and provide concrete examples. Note by passing that a similar representation theorem in a different framework was obtained in a recent preprint of Neshveyev and Tuset [27]. Amongst other applications, we establish a purely classical Ney-Spitzer theorem (Theorem 4.1) on the set of irreducible representation classes of the compact group $S U(n)$. The striking point of this result is that the proof intrinsically uses results of quantum probability theory.

Theorem 4.1 is also the starting point to an interesting counterpart of a result of [6]. On the group von Neumann algebra of $S U(n)$, the convolution operator by the normalized trace on the fundamental representation, leaves both the center of the von Neumann algebra and the von Neumann algebra of any maximal torus invariant. Therefore it induces two Markov chains on discrete spaces, and they are related by an $h$-transform [6]. This result turns out to have an analogue if one replaces $S U(n)$ by the quantum group $S U_{q}(n)$ of Woronowicz. In this case, classical results about combinatorics of representation of $S U_{q}(n)$, and the application of Theorem 4.1 results in Theorem 5.4.

This paper is organized as follows. In order to fix some notation, and for mathematical motivation, we start with a couple of reminders. In Section 2.1, we state the Ney-Spitzer theorem as we intend to generalize it. Then, in Section 2.2, we define the framework of Hopf algebras in which we study noncommutative probability theory. Section 3 is devoted to defining a quantum Martin boundary theory and showing that any positive harmonic element can be represented with respect to an adapted Martin kernel. By passing, we show that the minimal Martin boundary is isomorphic to a sphere under weak assumptions. In Section 4, we consider the restriction of a "Bernoulli" quantum walk to the center of the Hopf algebra and establish a Ney-Spitzer like theorem. Section 5 is an application of Section 4 to a quantum random walk on the dual of $S U_{q}(n)$ introduced by Izumi [18].

\section{Reminders of (non)commutative harmonic analysis}

\subsection{Classical Martin boundary}

Let $\mathcal{E}$ be a discrete countable state space and $P$ be a Markov (respectively sub-Markov) operator defined by $P f(x)=\sum_{y \in \mathcal{E}} P(x, y) f(y)$, where $P(x, y)$ is an array of positive real numbers assumed to satisfy $P 1=1$ (respectively $P 1 \leqslant 1$ ). In order to avoid technical difficulties, we assume that for each $x$, every $P(x, y)$ is zero except finitely many of them. Throughout the whole paper, we will identify, whenever relevant, the operator $P$ and the kernel $P(x, y)$. One defines inductively $P^{0}(x, y)=\delta_{x, y}, P^{n+1}(x, y)=\sum_{z \in \mathcal{E}} P^{n}(x, z) P(z, y)$ and the Green kernel $U(x, y)=\sum_{n \in \mathbb{N}} P^{n}(x, y)$. We make the usual assumptions of irreducibility and transience:

Assumption 2.1. For all $x, y \in \mathcal{E}$, one has $0<U(x, y)<\infty$.

A function $f$ is said to be harmonic with respect to $P$ if $P f=f$. The Martin kernel with a base point $x_{0}$ is defined as

$$
k(x, y)=U(x, y) / U\left(x_{0}, y\right) .
$$

Harnack inequalities imply that for all $x \in \mathcal{E}$, the function $k(x,$.$) is bounded. The Martin compactification MS of \mathcal{E}$ is defined as the smallest compact space in which $\mathcal{E}$ can be continuously and densely embedded and such that every 
function $k(x,$.$) can be uniquely extended by continuity to M S$. Let $M B$ be the boundary of $\mathcal{E}$ in $M S$. A positive harmonic function $f$ is said to be minimal if any harmonic function $g$ satisfying $0 \leqslant g \leqslant f$ is a multiple of $f$. There exists a measurable subset $M B^{\min }$ of $M B$ such that $x \in M B^{\min }$ if and only if $k(., x)$ is a positive minimal harmonic function.

Theorem 2.1. For every positive harmonic function $f$ satisfying $f(e)=1$, there exists a unique probability measure $\mu_{f}$ on $M B$ such that $\mu_{f}\left(M B^{\text {min }}\right)=1$ and for all $x \in \mathcal{E}, f(x)=\int_{\xi \in M B^{\min }} k(x, \xi) d \mu_{f}(\xi)$.

For the proof of the above theorem, see [29] or [20]. In view of this, it is natural to try to compute explicit examples. This turns out to be a difficult task, and one remarkable example of such a computation is done in [28].

Let $\mathcal{E}$ be the state space $\mathbb{Z}^{d}$, for some $d \geqslant 2$, and $\mu$ be a finitely supported measure (the hypothesis of finite support can be considerably weakened, but we do not enter into such technical considerations) whose mean on $\mathbb{R}^{d}$ is different from zero. We identify canonically $\mathbb{Z}^{d}$ with a lattice of the Euclidean space $\mathbb{R}^{d}$ with its scalar product $\langle.,$.$\rangle . Then, the set$

$$
E=\left\{x, \int_{\mathbb{Z}^{d}} \exp \langle x, X\rangle d \mu(X)=1\right\}
$$

is a $C^{\infty}$ submanifold of $\mathbb{R}^{d}$. It is diffeomorphic to the sphere $S^{d-1}$. It is a consequence on a theorem of Choquet and Deny (see [10]) that any positive function on $\mathbb{Z}^{d}$ harmonic with respect to the operator of convolution by $\mu$ (we call its operator $P_{\mu}$ ) admits an unique integral representation as a linear combination of functions $y \rightarrow e^{\langle x, y\rangle}$, $x \in E$.

Let $\beta$ be a continuous increasing bijection from $[0, \infty]$ to $[0,1]$. Let $\Pi$ be the map from $\mathbb{Z}^{d}$ to the canonical unit ball $B(0,1)$ of $\mathbb{R}^{d}$ given by $\Pi(x)=\beta(\|x\|) x /\|x\|$. This map is a topological injection, and the metrics it inherits gives rise to a compactification of $\mathbb{R}^{d}$ by $S^{d-1}$. The map $\Pi$ extends continuously to $\mathbb{Z}^{d} \cup S^{d-1}$ with value in $B(0,1)$. The map $u$ sending an element of $E$ to its normed outer normal vector is a $C^{\infty}$ diffeomorphism from $E$ to $S^{d-1}$.

Theorem 2.2 (Ney-Spitzer [28]). The Martin compactification of $\mathbb{Z}^{d}$ is $\mathbb{Z}^{d} \cup S^{d-1}$. The correspondence between $S^{d-1}$ and E arising from the Martin kernel is given by the map $u$. The extremal Martin boundary MB ${ }^{\text {min }}$ thus coincides with $M B$

Throughout the paper, we define

$$
\begin{aligned}
& \mathcal{H}_{\mathcal{E}, P}^{+}=\left\{f \in \mathbb{C}^{\mathcal{E}}, f \geqslant 0, P f=f, f(e)=1\right\}, \\
& \mathcal{H}_{\mathcal{E}, P}=\left\{f \in L^{\infty}(\mathcal{E}), \text { Pf }=f\right\} .
\end{aligned}
$$

The set $\mathcal{H}_{\mathcal{E}, P}^{+}$is convex compact for the topology of pointwise convergence. Therefore it admits extremal points. By definition, let $\mathcal{H}_{\mathcal{E}, P}^{+, e x}$ be this subset. By the Krein-Milman theorem, the closure of the convex hull of these extremal points is exactly $\mathcal{H}_{\mathcal{E}, P}^{+}$.

Thus, Theorem 2.1 identifies the extremal points of the above set with $M B^{\text {min }}$.

Remark 2.1. We shall say in this paper that we obtain a Ney-Spitzer like theorem when we give a compactification $M S=\mathcal{E} \cup M B$ of a state space, describe the extremal positive harmonic functions, describe a subset $M B^{\text {min }}$ of $M B$ and a bijection between $\mathcal{H}_{\mathcal{E}, P}^{+, e x}$ and $M B^{\text {min }}$. 


\subsection{Hopf algebras}

We fix some classical notations of operator algebra theory, and remind some elementary definitions and results of Hopf algebra theory.

Let $G$ be a topological compact group, and $d \mu$ its left and right invariant probability Haar measure. Let $L^{2}(G)$ be the $L^{2}$ space associated to this Haar measure. For $g \in G$, the unitary operator $\lambda_{g} \in B\left(L^{2}(G)\right)$ is defined by $\lambda_{g}: f \rightarrow\left(x \rightarrow f\left(g^{-1} x\right)\right)$. The mapping $g \rightarrow \lambda_{g}$ is continuous for the strong operator topology in $B\left(L^{2}(G)\right)$. The vector space $\operatorname{Vect}\left(\lambda_{g}, g \in G\right)$ is a $*$-subalgebra of $B\left(L^{2}(G)\right)$. Let $M(G)$ be the von Neumann algebra of $G$, i.e. the bicommutant of $\operatorname{Vect}\left(\lambda_{g}, g \in G\right)$ in $B\left(L^{2}(G)\right)$.

The set of equivalence classes of irreducible finite dimensional unitary representations of $G$ is denoted by $\Gamma$. For $x \in \Gamma, d_{x}$ is the corresponding dimension. By Peter-Weyl's theorem, we have the following isomorphism of von Neumann algebras:

$$
M(G) \cong \bigoplus_{x \in \Gamma} \mathbb{M}_{d_{x}}(\mathbb{C}) .
$$

The counit is the map $M(G) \rightarrow \mathbb{C}$, defined as the continuous linear extension of the map $\varepsilon\left(\lambda_{g}\right)=1$.

In the same way, the antipode is the continuous map $M(G) \rightarrow M(G)$ such that $S\left(\lambda_{g}\right)=\lambda_{g}$, and the coproduct is the map $M(G) \rightarrow M(G \times G) \cong M(G) \otimes M(G)$ such that $\widehat{\Delta}\left(\lambda_{g}\right)=\lambda_{g} \otimes \lambda_{g}$.

The quadruple $(M(G), \varepsilon, \widehat{\Delta}, S)$ is called the Hopf-von Neumann algebra of the group $G$.

For a von Neumann algebra $A$, we shall call $\widehat{A}$ the set of elements affiliated to $A$. By the Peter-Weyl theorem, $\widehat{M(G)}$ is a $*$-algebra endowed with a natural pointwise convergence topology. As a topological $*$-algebra, it is isomorphic to $\prod_{x \in \Gamma} \mathbb{M}_{d_{x}}(\mathbb{C})$.

One can see (see [4]), that $\widehat{\Delta}, \varepsilon$ and $S$ are also continuous for the topology of pointwise convergence, so that there is a unique way of extending them from $\widehat{M(G)}$ to $M(\widehat{G) \otimes M}(G)$ (respectively, $\widehat{M(G)}, \mathbb{C})$. According to Effros and Ruan (see [12]; also see [4]) we call this structure a topological $*$-Hopf algebra.

Let $M(G)$ be the set of finite rank operators in $M(G)$. A linear form $v: \widehat{M(G)} \rightarrow \mathbb{C}$ is said to be finitely supported iff it is continuous with respect to the pointwise convergence. Equivalently, there exists a faithful trace $\tau$ on $M(G)$ and an element $A \in M(G)$, such that for all $B \in \widehat{M(G)}$, one has $\mu(B)=\tau(A B)$. We denote by $(\widehat{M(G)})_{*}$ the vector space of finitely supported linear forms.

To summarize, we will be dealing with the following inclusions of algebras:

$$
M(G) \subset M(G) \subset \widehat{M(G)} .
$$

The first one is not a Hopf algebra, but the latter two are.

\subsection{Random walks and harmonic analysis}

We use the framework of Hopf algebras in order to define quantum random walks. Several groups of mathematicians have already inspected axiomatics (see [1]) and their properties (see for example $[3,19,30]$ ).

For $l \in \Gamma$, let $1_{l}$ be the minimal central idempotent in $M(G)$ associated to the irreducible representation $l \in \Gamma$ in $M(G)$. For $v$ and $\mu$ two states on $M(G)$, we define their convolution $\mu * v$ by the equation $\mu * v(f)=(\mu \otimes \nu) \widehat{\Delta}(f)$ for each $f \in M(G) . \mu * v$ is a state and we can define inductively $\nu^{* n}$ to be $\varepsilon$ if $n=0$ and $\nu * \nu^{* n-1}$ else. We define the operator $P_{\mu}$ on $M(G)$ by

$$
P_{\mu}(f)=(\mathrm{id} \otimes \mu) \widehat{\Delta}(f)
$$

and its iterates inductively by $P_{\mu}^{n}=$ id if $n=0$ and $P_{\mu}^{n}=P_{\mu} \circ P_{\mu}^{n-1}$ else. One has also $P_{\mu}^{n}=\left(\right.$ id $\left.\otimes \mu^{* n}\right) \widehat{\Delta}$.

The operator $P_{\mu}$ is the evolution operator associated to a quantum random walk on the dual of $G$ in the sense of [1]. It is a completely positive operator on $M(G)$. If $\mu\left(1_{l}\right)=0$ for any but finitely many $l$ 's, $P_{\mu}$ extends to a positive continuous operator on $\widehat{M(G)}$. 
An element $f$ in $\widehat{M(G)}$ is said to be harmonic with respect to $P_{\mu}$ iff

$$
P_{\mu} f=f .
$$

Biane showed in [4], that $\mathcal{H}_{P_{\mu}}^{+, e x}$ is the set

$$
E=\{f \in \widehat{M(G)}, f \geqslant 0, \widehat{\Delta} f=f \otimes f, \mu(f)=1\} .
$$

We shall say that an element $f \in \widehat{M(G)}$ such that $\widehat{\Delta} f=f \otimes f$ is an exponential, and call $\operatorname{Ex}(G)$ be the set of exponentials.

\section{Quantum Martin boundary theory}

\subsection{Representation of positive harmonic elements}

In this section, we define a Martin compactification and a Martin kernel, and show that every positive harmonic element can be represented by a state on the Martin boundary. For a completely positive continuous operator $Q$ from $M(G)$ into itself and $v$ a weight, $\nu Q$ is again a weight defined by $v Q f=v(Q(f))$. We write $v \leqslant \mu$ iff for any positive $f, v(f) \leqslant \mu(f)$. We need the following assumption:

Assumption 3.1. The weight $\mu$ is such that $\mu(1)=q \in(0,1)$ and that $\varepsilon U=\sum_{n \geqslant 0} \mu^{* n}$ is faithful. There exists a positive $A \in \operatorname{Ex}(G)$ and normal semifinite trace $\tilde{\mu}$ such that for any $f \in \widehat{M(G)}, \mu(f)=\tilde{\mu}(A f)$.

Let $U=\sum_{n \geqslant 0} P_{\mu}^{n}$. This operator has operator norm less than $(1-q)^{-1}$ and is the quantum analogue of the Green kernel.

We define the Martin Kernel to be the linear map

$$
K:(\widehat{M(G)})_{*} \rightarrow \widehat{M(G)}
$$

such that for any $v \in(\widehat{M(G)})_{*}, K_{v}$ satisfies for all $f \in M^{\circ}(G)$,

$$
\nu U(f)=\varepsilon U\left(A^{-1 / 2} K_{v} A^{1 / 2} f\right) .
$$

$K_{v}$ is well defined because the weight $\varepsilon U$ is faithful. A definition equivalent to this one has already appeared in P. Biane's papers (see [9]) in the context of $S U(2)$ for a tracial weight.

Lemma 3.1. Let $g \in M(G)$. Then one has $g=U\left(g-P_{\mu}(g)\right)$.

Proof. It is enough to remark that $U f$ is defined for any $f \in M(G)$, that under the Assumption 3.1 it is the norm limit of $\sum_{k=0}^{n} P_{\mu}^{k} f$, and that $\sum_{k=0}^{n} P_{\mu}^{k}\left(f-P_{\mu} f\right)=f-P_{\mu}^{n+1} f$. But $P_{\mu}^{n+1} f$ has norm tending towards zero as $n$ tends to infinity.

\section{Proposition 3.2.}

- $K$ is positive, injective, and its image is contained in $M(G)$.

- The norm closure of $\operatorname{span}\left\{K_{v}, v \in(\widehat{M(G)})_{*}\right\}$ contains the $C^{*}$-algebra $\mathcal{K}$ of compact operators in $M(G)$.

Proof. It is known that $\phi: \mathbb{M}_{n}(\mathbb{C}) \rightarrow \mathbb{C}$ is a weight iff there exists a positive matrix $B$ such that for all $A$, $\phi(A)=\operatorname{Tr}(A B)$. Therefore, since the weight $\varepsilon U$ satisfies Assumption 3.1, if $v$ is a weight then $A^{1 / 2} K_{v} A^{1 / 2}$ is positive. Since $A$ is invertible, $K_{v}$ is also positive. 
For $v$ a finitely supported weight, there exists by Assumption 3.1 an integer $n$ and a constant $\alpha$ such that $v \leqslant \alpha \sum_{i=0}^{n} \varepsilon P_{\mu}^{i}$. By positivity of $P_{\mu}$ this implies that $\nu U \leqslant(n+1) \alpha \varepsilon U$. This implies that $K_{v}$ is bounded.

If $K_{v}=0$, then by faithfulness of $\varepsilon U$, one has $v U=0$, and by Lemma 3.1, $v=0$, which proves the "into".

For the second statement, it is enough to show that for every positive finite dimensional operator $f \in \widehat{M(G)}$, there exists $v$ such that $K_{v}=f$.

Let $v$ be the linear form such that for any $g \in M(G)$,

$$
v(g)=\varepsilon U\left(A^{-1 / 2} f A^{1 / 2}\left(g-P_{\mu} g\right)\right) .
$$

Then $v$ is finitely supported, so that $K_{v}$ is well defined; and one can check using Lemma 3.1 that it satisfies $K_{v}=f$.

We define the Martin space $M S$ to be the $C^{*}$-algebra

$$
M S=C^{*}\left(K_{v}, v \in(\widehat{M(G)})_{*}\right) .
$$

By Proposition $3.2, \mathcal{K}$ is a subspace of $M S$, and therefore an ideal. Let the Martin boundary be the $C^{*}$-algebra $M B=M S / \mathcal{K}$. The Martin compactification of the dual $\widehat{G}$ of $G$ is then defined to be the following exact sequence:

$$
0 \rightarrow \mathcal{K} \rightarrow M S \rightarrow M B \rightarrow 0
$$

\section{Remark 3.1.}

- The exact sequence (3.3) yields an identification of the state space of $M B$ with the subset of states of $M S$ that vanish on $\mathcal{K}$. We shall make this identification without mention whenever relevant in the remainder of this paper.

- In Sections 4 and 5, we shall restrict to commutative examples and make an abuse of notation: we will denote by $M S$ and $M B$ the topological underlying spaces in the compactification, instead of the $C^{*}$ algebra of continuous functions on these spaces.

\section{Theorem 3.1.}

- For each positive harmonic element $h$ in $\widehat{M(G)}$ there exists a state $\phi_{h}$ on $M B$ such that for every finitely supported linear form $v$ on $M(G)$, one has $v(h)=\phi_{h}\left(K_{v}\right)$.

- This representation is unique if $\operatorname{span}\left\{K_{\nu}, v \in(\widehat{M(G)})_{*}\right\}$ is dense in MS.

Proof. If $\phi$ is a state on $M B$ then the element $h_{\phi} \in \widehat{M(G)}$ defined by $v\left(h_{\phi}\right)=\phi\left(K_{v}\right)$ for all $v \in(\widehat{M(G)})_{*}$, is positive. Let us first show that it is harmonic.

We need to show that $P_{\mu} h_{\phi}=h_{\phi}$, or equivalently, that for all $v \in(\widehat{M(G)})_{*}, v\left(P_{\mu} h_{\phi}\right)=v\left(h_{\phi}\right)$. But $v P_{\mu} h_{\phi}=$ $(\mu * v) h_{\phi}=\phi\left(K_{\mu * v}\right)$ and $v\left(h_{\phi}\right)=\phi\left(K_{v}\right)$, therefore it is enough to show that $\phi\left(K_{\mu * v}\right)=\phi\left(K_{v}\right)$.

We have, for all $f \in \widehat{M(G)}$

$$
\varepsilon U\left(A^{-1 / 2}\left(K_{\mu * \nu}-K_{\nu}\right) f A^{1 / 2}\right)=(\mu * v-v) U f=v f
$$

(second equality arises from Lemma 3.1). But since $v \in(\widehat{M(G)})_{*}$, this implies that $K_{\mu * v}-K_{v}$ has finite rank, thus is compact. Therefore $\phi\left(K_{\mu * \nu}-K_{\nu}\right)=0$.

Furthermore, $h_{\phi}$ satisfies $\varepsilon h_{\phi}=1$ and one sees that the linear map

$$
\Xi:(M S)^{*} \rightarrow \widehat{M(G)}
$$

that maps $\phi$ to $h_{\phi}$ is continuous for the pointwise convergence topology. 
We will now show that for every extremal harmonic element $h$, there exists a weight $\phi$ on $M S$ such that for any $v \in(\widehat{M(G)})_{*}$, one has

$$
v(h)=\phi\left(K_{v}\right) \text {. }
$$

Since $\Xi$ is linear, any convex combination of extremal harmonic elements can be represented. Furthermore, $\Xi$ is continuous, therefore, any harmonic element that can be approximated in the pointwise convergence topology by a convex combination of extremal harmonic elements can be represented, therefore, any element can be represented.

Let $h$ be such a minimal harmonic element. By Biane's theorem [4], it satisfies $\widehat{\Delta} h=h \otimes h$ and $\mu(h)=1$. Let $\widehat{M(h)}$ be the closure in $\widehat{M(G)}$ of the algebra generated by $h$. This is obviously a topological Hopf $*$-subalgebra of $\widehat{M(G)}$. This algebra is commutative, cocommutative and closed, therefore one can show directly that the operations inf and sup are well defined inside this algebra.

In $\widehat{M(h)}$, consider a sequence $h_{k}$ of positive elements such that $U h_{k}$ tends and increases towards $h$ as $k$ goes towards infinity. The existence of such a sequence is a consequence of standard probabilistic considerations, but we justify it nonetheless.

Let $f_{k}=\inf (k I d, h)$, where the infimum is taken on the commutative affiliated algebra $\widehat{M(h)} \cdot f_{k}$ is bounded and satisfies $P_{\mu}\left(f_{k}\right) \leqslant f_{k}$. Let $h_{k}=f_{k}-P f_{k}$. This element is positive and it can not be zero because $h$ is extremal and non-bounded, thus $f_{k}$ would have to be a multiple of $h$, which would result in $h=0$. By Lemma 3.1 this implies that $f_{k}=U h_{k}$. Last, it is obvious that $f_{k}$ tends towards $h$ in the pointwise convergence topology, as $k \rightarrow \infty$.

Consider $\phi_{k}=\varepsilon U\left(A^{-1 / 2} h_{k} A^{1 / 2} \cdot\right)$. It is a state on the norm closed operator system generated by $K_{\mu}$, and it satisfies $\phi_{k}(1)=\varepsilon U h_{k} \leqslant 1$. By a classical result (see [11], p. 50, Lemme 2.10.1), it extends to a state on $M S$.

Furthermore, $\phi_{k}\left(K_{v}\right)=v\left(U h_{k}\right)$ tends towards $v(h)$ as $k$ tends towards infinity. This proves that there exists a cluster point $\phi$ of the sequence $\left\{\phi_{k}\right\}$ in the weak $*$ topology such that $\phi\left(K_{v}\right)=v(h)$. This state vanishes on finite operators, therefore on compact operators. So is actually a state of $M B$.

\subsection{Topological structure of the boundary}

In this section, we assume that $G$ is a compact simply connected Lie subgroup of $\mathbb{U}_{n}(\mathbb{C})$ with Lie algebra $\mathfrak{g}$. Let $\mathfrak{g}_{\mathbb{C}}$ be the complexified Lie algebra, $G_{\mathbb{C}}$ be the complexified Lie group, and $(\rho, V)$ be the fundamental representation of $G$.

The left regular representation yields an identification of $\mathfrak{g}_{\mathbb{C}}$ with a Lie subalgebra of $\widehat{M(G)}$. If $f \in \mathfrak{g}_{\mathbb{C}}$, then $\widehat{\Delta} f=f \otimes 1+1 \otimes f$. The map $E X P: \widehat{M(G)} \rightarrow \widehat{M(G)}$ defined by the usual series is such that for any $f \in \mathfrak{g}_{\mathbb{C}}$, $\widehat{\triangle E X P f}=E X P f \otimes E X P f$.

By a slight modification of a result of Biane ([4], Proposition 11 and Lemme 12), if any irreducible representation of $G$ is contained in some tensor power of $V$, then the set of non-zero exponentials in $\widehat{M(G)}$

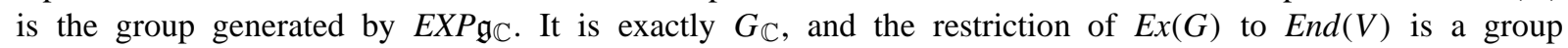
isomorphism between $\operatorname{Ex}(G)$ and $G_{\mathbb{C}}$. An explicit isomorphism is obtained by restricting $\operatorname{Ex}(G)$ to the fundamental representation. We call

$$
i: G_{\mathbb{C}} \rightarrow \operatorname{Ex}(G)
$$

the converse of this isomorphism.

The following theorem answers a question raised by Biane about the topology of the boundary.

Theorem 3.2. Let $\mu$ be a weight on $M(G)$ satisfying $\mu(1)=q \in(0,1)$ and Assumption 3.1.

Then, the set $H_{P_{\mu}}^{+, \text {ex }}$ of extremal harmonic elements is diffeomorphic to the sphere $S^{k-1}$, where $k$ is the dimension of the Lie algebra $\mathfrak{g}$. 
Proof. Let $\mathfrak{g}_{s a}$ be the real vector subspace of $\widehat{M(G)}$ of self adjoint elements of $\mathfrak{g}_{\mathbb{C}}$ in $\widehat{M(G)}$. Let $x \in \mathfrak{g}_{s a}$ be non-zero, and $f_{x}$ the map $\mathbb{R} \rightarrow \mathbb{R}$ given by $f_{x}(t)=v(E X P t x)$. This map is always positive. Since $\operatorname{tr} x=0$ and $x$ is Hermitian, it has one negative eigenvalue and one positive eigenvalue. Therefore $\lim _{ \pm \infty} f_{x}=\infty$. Besides by definition, $f_{x}(0)=q<1$. The function $f_{x}$ admits the second derivate $v\left(x^{2}\right.$ EXPtx) at $t$, therefore it is positive. Thus, the function $f_{x}$ is convex; therefore there exists only two real numbers $t_{x}^{+}$(respectively $t_{x}^{-}$) satisfying $t_{x}^{+}>0$ and $f_{x}\left(t_{x}^{+}\right)=1$ (respectively $t_{x}^{-}<0$ and $f_{x}\left(t_{x}^{-}=1\right)$ ). But the map EXP is a diffeomorphism from $\mathfrak{g}_{s a}$ onto $E=\{x \in \widehat{M(G)}, x \geqslant 0, \widehat{\Delta} x=x \otimes x\}$. Therefore the inverse image of $\mathcal{H}_{P}^{+, \text {ex }}$ under exp is a closed star-like subset around 0 , therefore it is homeomorphic to the sphere $S^{k-1}$.

\section{A Ney-Spitzer theorem for a random walk on a Weyl chamber}

It would be interesting and seems challenging to obtain nice generalizations of the result of [9] in the framework developped above. We are not able to perform fully such computations. Yet, it is possible to obtain a Ney-Spitzer like theorem if one restrict a tracial quantum random walk on $S U(n)$ to the center of its Hopf algebra. In this section, we establish a Ney-Spitzer like theorem for the most elementary quantum random walk, improving previous results of [6].

\subsection{Main result}

In the Euclidean space $\mathbb{R}^{n}, n \geqslant 3$, with canonical basis $\left(\tilde{e}_{i}\right)_{i=1}^{n}$, we consider the lattice $L$ spanned by $e_{i}=\tilde{e_{i}}-\left(\tilde{e_{1}}+\cdots+\tilde{e_{n}}\right) / n$. There is a unique way to write $x \in L$ under the form $x=\sum_{i=1}^{n} x_{i} e_{i}$ such that every $x_{i} \in \mathbb{N}$ and one at least is zero. We call $\left(x_{1}, \ldots, x_{n}\right)$ the coordinates of $x$ and $\sum x_{i}=|x|$ the length of $x$. Let

$$
\begin{aligned}
& W_{n}=\left\{x \in L, x_{1} \geqslant x_{2} \geqslant \cdots \geqslant x_{n}=0\right\}, \\
& \stackrel{\circ}{W}_{n}=\left\{x \in L, x_{1}>x_{2}>\cdots>x_{n}=0\right\} .
\end{aligned}
$$

The lattice $L$ is compactified by the sphere $S^{n-2}$ in the following sense: a sequence $x^{d}$ of $L$ tends towards $y \in S^{n-2}$ iff its Euclidean norm $\left\|x_{n}\right\|$ tends to infinity and $x^{d} /\left\|x^{d}\right\| \rightarrow y$. Consider the simplex

$$
\Sigma=\left\{y^{\prime}=\left(y_{1}^{\prime}, \ldots, y_{n}^{\prime}\right): y_{1}^{\prime} \geqslant \cdots \geqslant y_{n}^{\prime}=0, \sum_{i} y_{i}^{\prime}=1\right\} \subset \mathbb{R}^{n} .
$$

We embed it into $S^{n-2}$ by the map $y^{\prime} \rightarrow y^{\prime} /\left\|y^{\prime}\right\|$. By doing so, the above compactification induces a compactification of $\stackrel{\circ}{W}_{n}$ by $\Sigma$.

Namely, let $y^{d}=\left(y_{1}^{d}>y_{2}^{d}>\cdots>y_{n}^{d}=0\right)_{d \geqslant 0}$ be a series of elements of $\stackrel{\circ}{W}_{n}$. Then it converges iff $y_{d}$ is constant for $d$ large enough or if $\left|y_{d}\right|$ tends towards infinity and for all $i, y_{i}^{d} /\left|y^{d}\right|$ admits a limit $y_{i}^{\prime}$.

Let $0<q<1$ be a real number. Consider the measure

$$
\mu=\sum_{i=1}^{n} \frac{q}{n} \delta_{e_{i}}
$$

where $\delta_{x}$ is the Dirac mass at $x$.

The Martin theory with respect to $P_{\mu}$ on the state space $L$ is completely understood, and stated by Theorem 2.2.

Let us now define a new random walk on the state space $\stackrel{\circ}{W}_{n}$. The vector $\rho=(n-1, n-2, \ldots, 0)$ is such that $\stackrel{\circ}{W}_{n}=\rho+W_{n}$. In the sequel, we abbreviate $P_{\mu}$ by $P$. Our random walk in $\mathscr{W}_{n}$ is obtained from $P$ and conditioned not to hit $\partial W_{n}=W_{n}-\stackrel{\circ}{W}_{n}$. Call $\stackrel{\circ}{P}$ its transition kernel. For $x \in \stackrel{\circ}{W}_{n}$, its transition kernel satisfies

$$
\stackrel{\circ}{P}(x, y)= \begin{cases}q / n & \text { iff } y-x=e_{i} \text { and } y \in \stackrel{\circ}{W}_{n}, \\ 0 & \text { otherwise. }\end{cases}
$$


The main result of this section is the following Ney-Spitzer like theorem (in the sense of Remark 2.1).

Theorem 4.1. The Martin boundary associated to the sub-Markov kernel $\stackrel{\circ}{P}$ on the state space $\dot{W}_{n}$ with respect to the base point $\rho$, is homeomorphic to $\Sigma$. Furthermore, $M B=M B^{\mathrm{min}}$.

The remainder of this section is devoted to proving this theorem. In Section 4.2, we compute MB. In Section 4.3, we consider $\stackrel{\circ}{W}_{n}$ as a canonical basis for the fusion algebra of $S U(n)$, and consider the Markov operator $\widetilde{P}$ obtained by considering the convolution by the normalized fundamental representation. Thanks to a result of Biane, its abstract Martin boundary can be computed and identified with that of $\stackrel{\circ}{P}$. We use that to show that $M B=M B^{\min }$.

\subsection{Asymptotics of the Martin kernel}

The translation invariance of $P$ implies that we can define for any $l$ the one variable functions

$$
P^{l}(y-x)=P_{\mu}^{l}(x, y), \quad U(y-x)=U(x, y) .
$$

From now on, we take the convention that for $x \in \mathbb{N}$,

$$
x !^{-1}=0 \quad \text { if } x<0 .
$$

Lemma 4.2. The following holds true:

$$
\begin{aligned}
& P^{l}(y)= \begin{cases}\frac{(|y|+k n) !}{\prod_{i=1}^{n}\left(y_{i}+k\right) !}(q / n)^{l} & \text { if } \exists k \in \mathbb{N}, l=k n+|y|, \\
0 & \text { otherwise, }\end{cases} \\
& \stackrel{\circ}{P}^{l}(x, y)= \begin{cases}l !(q / n)^{l} \operatorname{det}\left(y_{i}-x_{j}+k\right) !^{-1} & \text { if } \exists k \in \mathbb{N}, l=k n+|y|-|x|, \\
0 & \text { otherwise. }\end{cases}
\end{aligned}
$$

Proof. The first point is elementary combinatorics. For the second one, if $x=\left(x_{1}, \ldots, x_{n}\right)$, then for $\sigma$ a permutation of $[1, n]$, let $x_{\sigma}=\left(x_{\sigma(1)}, \ldots, x_{\sigma(n)}\right)$. We have, by the reflexion principle (see for example [6], Lemma 4.1)

$$
\stackrel{\circ}{P}^{l}(x, y)=\sum_{\sigma \in \mathcal{S}_{n}} P_{\mu}^{l}\left(x_{\sigma}, y\right) \varepsilon(\sigma)
$$

where $\varepsilon(\sigma)$ is the signature of the permutation $\sigma$, and the result follows together with the fact that $|y-x|=$ $|y|-|x| \bmod n$.

Let $Y=\left(Y_{1}, \ldots, Y_{n}\right)$ be an $n$-tuple of formal variables. Recall that the Vandermonde determinant is the polynomial $V(Y)=\prod_{1 \leqslant i<j \leqslant n}\left(Y_{j}-Y_{i}\right)$. Let $x \in W$. The function

$$
s_{x}\left(Y_{i}\right)=\frac{\operatorname{det}\left(Y_{i}^{x_{j}+n-j}\right)}{V\left(Y_{i}\right)}
$$

is a symmetric polynomial in $Y$ homogeneous of degree $|x|$. It is known as the Schur polynomial (see [13]). It is classical (see [14]) that $W_{n}$ is in one to one correspondence with the set of classes of irreducible representations of $S U(n)$ up to isomorphism. By Weyl character formula, $s_{x}$ is known to be the character of the irreducible representation associated to $x$ evaluated on $\operatorname{diag}\left(Y_{1}, \ldots, Y_{n}\right)$.

Lemma 4.3. Let $x, y \in \stackrel{\circ}{W}_{n}$ and let $k, l \in \mathbb{N}$ satisfying $l=k n+|y|-|x|$. Set

$$
f(x, y, l)=\frac{(k n+|y|-|x|) !}{(k n+|y|) !} .
$$


Then

$$
\stackrel{\circ}{P}^{l}(x, y)=(q / n)^{-|x|} V\left(y_{i}\right) f(x, y, l) P^{l+|x|}(y) s_{x-\rho}\left(y_{i}+k\right)(1+\mathrm{o}(1)),
$$

where the symbol o(1) has to be understood as $|y| \rightarrow \infty$, uniformly in $k, k^{2} \geqslant|y|$.

Proof. Since by Lemma 4.2

$$
\stackrel{\circ}{P}^{l}(x, y)=l !(q / n){ }^{l} \operatorname{det}\left(y_{i}-x_{j}+k\right) !^{-1}
$$

one has

$$
\stackrel{\circ}{P}^{l}(x, y) / P^{l+|x|}(y)=(q / n)^{-|x|} f(x, y, l) \operatorname{det}\left(\frac{\left(y_{i}+k\right) !}{\left(y_{i}-x_{j}+k\right) !}\right) .
$$

The expression $\left(y_{i}+k\right) ! /\left(y_{i}-x_{j}+k\right)$ ! is a polynomial in the variable $y_{i}+k$ whose leading term is $\left(y_{i}+k\right)^{x_{j}}$. By multilinearity of the determinant and the definition of Schur polynomials this implies that

$$
\operatorname{det}\left(\frac{\left(y_{i}+k\right) !}{\left(y_{i}-x_{j}+k\right) !}\right)=V(y) s_{x-\rho}\left(y_{i}+k\right)(1+\mathrm{o}(1)) .
$$

Let

$$
A_{q}=\left\{y^{\prime \prime}=\left(y_{1}^{\prime \prime} \geqslant \cdots \geqslant y_{n}^{\prime \prime} \geqslant 0\right), \prod_{i=1}^{n} y_{i}^{\prime \prime}=1, \sum_{i=1}^{n} y_{i}^{\prime \prime}=n q^{-1}\right\} .
$$

To $y^{\prime} \in \Sigma$ we associate an element $y^{\prime \prime}=\phi\left(y^{\prime}\right) \in A_{q}$ defined by $y_{i}^{\prime \prime}=q^{-1} n\left(y_{i}^{\prime}+\alpha\right) /(1+n \alpha)$ where $\alpha$ is the only real number such that $\prod_{i=1}^{n}\left(y_{i}^{\prime}+\alpha\right) /(1+n \alpha)=(q / n)^{n}$. The fact that this map is well defined (i.e. the fact that the real number $\alpha$ is unique) is a consequence of the proof of Lemma 4.5, in which it is showed that $\alpha \rightarrow \prod_{i=1}^{n}\left(y_{i}^{\prime}+\alpha\right) /(1+n \alpha)$ is non-decreasing. The fact that it is continuous is a consequence of the continuity of the roots of a polynomial with respect to its coefficients.

Obviously, the vector $\left(y_{1}^{\prime \prime}-y_{n}^{\prime \prime}, \ldots, y_{n-1}^{\prime \prime}-y_{n}^{\prime \prime}, 0\right)$ is proportional to $\left(y_{1}^{\prime}, \ldots, y_{n}^{\prime}\right)$, therefore, the continuous map that, to an element of $y^{\prime \prime}$ of $A_{q}$ associates $y^{\prime}$ defined by $y_{i}^{\prime}=\left(y_{i}^{\prime \prime}-y_{n}^{\prime \prime}\right) /\left(\sum_{j} y_{j}^{\prime \prime}-y_{n}^{\prime \prime}\right)$, is a left and right inverse of $\phi$. As a summary, we have

Lemma 4.4. The map $\phi: \Sigma \rightarrow A_{q}$ is a homeomorphism.

The key to the proof of the main result of this section is a precise understanding of the asymptotics of the summands of the kernel $u$. In the remainder of the section, we make the following

Assumption 4.1. The sequence $\left(y^{d}\right)_{d \in \mathbb{N}}, y^{d} \in \stackrel{\circ}{W}_{n}$ admits a limit in the boundary $\Sigma$ of $\stackrel{\circ}{W}_{n}$ defined at Eq. (4.2).

Lemma 4.5. For all $d$ it is possible to choose two integers $a_{d}<b_{d}$ such that the functions $P$ and $U$ defined at Eq. (4.3) satisfy:

$$
\begin{aligned}
& U\left(y^{d}\right) \sim \sum_{k=a_{d}}^{b_{d}} P^{\left|y_{d}\right|+k n}\left(y^{d}\right), \\
& (q / n)^{n}\left(\left|y^{d}\right|+k n\right)^{n} \prod_{i=1}^{n}\left(y_{i}^{d}+k\right)^{-1} \sim 1
\end{aligned}
$$

uniformly in $k \in\left[a_{d}, b_{d}\right]$ as $d \rightarrow \infty$. 
Proof. Let

$$
f_{k}(y)=P^{|y|+(k+1) n}(y) / P^{|y|+k n}(y)=(q / n)^{n} \frac{(|y|+(k+1) n) !}{(|y|+k n) ! \prod_{i=1}^{n}\left(y_{i}+k+1\right)} .
$$

This function is defined a priori only for $k \in \mathbb{N}^{*}$, but it admits a natural extension on the index set $k \in \mathbb{R}_{+}^{*}$ because it is a real rational fraction in $k$ with no poles in $\mathbb{R}_{+}^{*}$.

As $y^{d} \rightarrow y^{\prime}$, the function $g_{d}: t \rightarrow f_{t\left|y^{d}\right|}\left(y^{d}\right)$ converges pointwise on $(0, \infty)$ towards

$$
g_{\infty}: t \rightarrow \frac{q^{n}(1 / n+t)^{n}}{\prod_{i=1}^{n}\left(y_{i}^{\prime}+t\right)} .
$$

This function is strictly decreasing. Indeed, its logarithmic derivative is

$$
\frac{g_{\infty}^{\prime}}{g_{\infty}}: t \rightarrow \frac{n}{1 / n+t}-\sum_{i=1}^{n} \frac{1}{y_{i}^{\prime}+t}
$$

and the inequality between harmonic mean and natural mean implies that this logarithmic derivative is always $<0$.

Let $[a, b]$ be a closed subinterval of $(0, \infty)$. For $d$ large enough, $t \rightarrow g_{d}(t)$ admits a logarithmic derivative that is nonpositive everywhere on $[a, b]$. Indeed, the function

$$
(t, d) \rightarrow \frac{\partial}{\partial t} \log g_{d}(t)
$$

is easily seen to be a continuous function on the set $[a, b] \times(\mathbb{N} \cup\{+\infty\})$. Therefore for $d$ large enough, $g_{d}$ is nonincreasing. This implies that the convergence of $g_{d}$ towards $g_{\infty}$ holds uniformly on compact subsets of $(0, \infty)$.

Let $\varepsilon_{d}=\left|y_{d}\right|^{-1 / 3}$ and let $\left[a_{d}, b_{d}\right]$ be the greatest interval such that $\left|f_{k}\left(y_{d}\right)-1\right| \leqslant \varepsilon_{d}$ for all $k \in\left[a_{d}, b_{d}\right]$. By the property of uniform convergence on compact subsets and the fact that the limit is non-increasing, this interval is well defined (i.e. non-empty) for $d$ large enough and there exist non-negative constants $C_{1}$ and $C_{2}$ depending only on $n$ such that $C_{1}\left|y^{d}\right|^{2 / 3} \leqslant\left|b_{d}-a_{d}\right| \leqslant C_{2}\left|y^{d}\right|^{2 / 3}$. We show further down in this proof that $b_{d}-a_{d}$ is actually of the order of $\left|y^{d}\right|^{2 / 3}$ and therefore is non-zero for $\left|y^{d}\right|$ large enough. For $k \in\left[0, a_{d}-1\right]$, we have

$$
P^{\left|y_{d}\right|+k n+n}\left(y^{d}\right) / P^{\left|y_{d}\right|+k n}\left(y^{d}\right) \geqslant 1+\left|y_{d}\right|^{-1 / 3}
$$

and for $k \geqslant b_{d}$,

$$
P^{\left|y_{d}\right|+k n+n}\left(y^{d}\right) / P^{\left|y_{d}\right|+k n}\left(y^{d}\right) \leqslant 1-\left|y_{d}\right|^{-1 / 3} .
$$

An immediate recursion together with a geometric series summation argument shows that

$$
\sum_{k=0}^{a_{d}-1} P^{\left|y_{d}\right|+k n}\left(y^{d}\right) \leqslant P^{\left|y_{d}\right|+a_{d} n}\left(y^{d}\right)\left|y_{d}\right|^{1 / 3}
$$

and

$$
\sum_{k \geqslant b_{d}} P^{\left|y_{d}\right|+k n}\left(y^{d}\right) \leqslant P^{\left|y_{d}\right|+b_{d} n}\left(y^{d}\right)\left|y_{d}\right|^{1 / 3} .
$$

Let $\left[a_{d}^{1}, b_{d}^{1}\right]$ (respectively $\left[a_{d}^{2}, b_{d}^{2}\right]$ ) be the greatest interval such that $f_{k}\left(y_{d}\right) \in\left[1,1+\varepsilon_{d}\right]$ (respectively $f_{k}\left(y_{d}\right) \in$ $\left.\left[1-\varepsilon_{d}, 1\right]\right)$. There is a non-negative constant $C_{3}$ such that $\left[a_{d}^{1}, b_{d}^{1}\right]$ and $\left[a_{d}^{2}, b_{d}^{2}\right]$ are of length more than $C_{3}\left|y^{d}\right|^{2 / 3}$. Furthermore, by the definition of $f_{k}$ there exists an index $i \in\{1,2\}$ such that for any $k \in\left[a_{d}^{i}, b_{d}^{i}\right]$,

$$
P^{\left|y_{d}\right|+k n}\left(y^{d}\right) \geqslant \max \left\{P^{\left|y_{d}\right|+a_{d} n}\left(y^{d}\right), P^{\left|y_{d}\right|+b_{d} n}\left(y^{d}\right)\right\} .
$$

This shows that $U\left(y^{d}\right) \sim \sum_{k=a_{d}}^{b_{d}} P^{\left|y_{d}\right|+k n}\left(y^{d}\right)$. The second point, i.e. the asymptotics $(q / n)^{n}\left(\left|y^{d}\right|+k n\right)^{n} \times$ $\prod_{i=1}^{n}\left(y_{i}^{d}+k\right)^{-1} \sim 1$ is immediate from the definition of the set $[a, b]$. 
Proposition 4.6. Let $\left(y^{d}\right)_{d \in \mathbb{N}}$ be a sequence of $\stackrel{\circ}{W}_{n}$ converging towards an element $y^{\prime} \in \Sigma$, following Assumption 4.1. Then

$$
\lim _{d} \frac{\stackrel{\circ}{U}\left(x, y^{d}\right)}{U}=s_{x-\rho}\left(y_{i}^{\prime \prime}\right)
$$

where $y^{\prime \prime}=\phi\left(y^{\prime}\right)$, as defined in Lemma 4.4 .

Proof. Fix $x \in \stackrel{\circ}{W}_{n}$. One has

$$
\stackrel{\circ}{U}\left(x, y^{d}\right)=\sum_{k=0}^{\infty} \stackrel{\circ}{P}^{n k+\left|y^{d}-x\right|}\left(x, y^{d}\right) .
$$

For $y^{d}$, introduce the integers $a_{d}, b_{d}$ constructed in Lemma 4.5. By construction of these integers, the function $f$ introduced in Lemma 4.3 is such that the sequences

$$
\max \left\{f\left(x, y^{d},\left|y^{d}-x\right|+k n\right) / f\left(x, y^{d},\left|y^{d}-x\right|+a_{d} n\right), k \in\left[a_{d}, b_{d}\right]\right\}
$$

and

$$
\min \left\{f\left(x, y^{d},\left|y^{d}-x\right|+k n\right) / f\left(x, y^{d},\left|y^{d}-x\right|+a_{d} n\right), k \in\left[a_{d}, b_{d}\right]\right\}
$$

tend to one as $d \rightarrow \infty$. Therefore, for $d$ large enough,

$$
\sum_{k=a_{d}}^{b_{d}} \stackrel{\circ}{P}^{n k+\left|y^{d}\right|-|x|}\left(x, y^{d}\right) \sim(q / n)^{-|x|} f\left(x, y^{d},\left|y^{d}\right|-|x|+a_{d} n\right) s_{x-\rho}\left(y_{i}+a_{d}\right) \sum_{k=a_{d}}^{b_{d}} P^{n k+\left|y^{d}\right|}\left(y^{d}\right) .
$$

The inequality $0 \leqslant \stackrel{\circ}{P}^{l}(x, y) \leqslant P^{l}(x, y)$ and Lemma 4.5 thus imply that

$$
\stackrel{\circ}{U}\left(x, y^{d}\right) \sim(q / n)^{-|x|} f\left(x, y^{d},\left|y^{d}\right|-|x|+a_{d} n\right) s_{x-\rho}\left(y_{i}+a_{d}\right) \sum_{k=a_{d}}^{b_{d}} P^{n k+\left|y^{d}\right|}\left(y^{d}\right)
$$

as $d$ tends to infinity. Equivalently, in the particular case of $x=\rho$, we have

$$
\stackrel{\circ}{U}\left(x, y^{d}\right) \sim(q / n)^{-|\rho|} f\left(\rho, y^{d},\left|y^{d}\right|-|\rho|+a_{d} n\right) \sum_{k=a_{d}}^{b_{d}} P^{n k+\left|y^{d}\right|}\left(y^{d}\right) .
$$

Taking the quotient of (4.6) and (4.7) together with the definition of $y^{\prime \prime}$ in Lemma 4.4 imply that

$$
\frac{\stackrel{\circ}{U}\left(x, y^{d}\right)}{\stackrel{\circ}{U}\left(\rho, y^{d}\right)} \sim s_{x-\rho}\left(y_{i}^{\prime \prime}\right) \text {. }
$$

Proof of first part of Theorem 4.1. Computation of $M S$. Proposition 4.6 shows that the compactification of $W_{n}$ by $\Sigma$ is greater than the Martin boundary, in the following sense: there exists a continuous map $\pi$ of $\stackrel{\circ}{W}_{n} \cup \Sigma$ onto $M S$ whose restriction to $\stackrel{\circ}{W}_{n}$ is the identity. The map $\pi$ is actually a bijection. Indeed, let $y, z$ be two different elements of $A_{q}$. Then by a standard Fourier transform argument, there exists a representation $x$ such that $s_{x}\left(y_{i}\right) \neq s_{x}\left(z_{i}\right)$, and the map $\pi$ is into.

In particular, this proof shows that minimal harmonic functions are of the kind $x \rightarrow s_{x-\rho}\left(y_{i}^{\prime \prime}\right)$, where $y_{i}^{\prime \prime} \in A_{q}$. The forthcoming section is devoted to proving that all these functions are indeed minimal. 


\subsection{Extremal Martin boundary}

This part is devoted to computing $M B^{\text {min }}$, and showing that $M B^{\text {min }}=M B$. Let $\mathfrak{s l}_{n}$ be the complex Lie algebra of $S L(n)$.

$S U(n)$ admits a natural left action by conjugation on $\widehat{M(S U(n)})$, which we denote by Ad. The normalized trace $\operatorname{tr}$ of the fundamental representation extends by linearity and continuity to a positive linear functional on $M \widehat{(S U(n))}$. This allows to define the positive convolution operator

$$
\widetilde{P}=q(\operatorname{tr} \otimes \mathrm{id}) \widehat{\Delta} \text {. }
$$

Let $Z \widehat{(S U(n)})$ be the center of $\widehat{M(S U(n)})$. Since tr is invariant under the adjoint action, Ad and $\widetilde{P}$ commute. The algebra $Z \widehat{(S U(n)})$ is the fixed point algebra of Ad, therefore $\widetilde{P}$ leaves $\overline{Z(S U(n))}$ invariant and defines a new submarkovian operator on $\stackrel{\circ}{W}_{n}$ (upon the obvious identification of $\mathbb{C}^{\mathscr{W}_{n}}$ with $Z \widehat{(S U(n))}$ ).

It turns out that there is a link between the restriction of $\widetilde{P}$ and $\stackrel{\circ}{P}$, namely

Theorem 4.7 ([6], Theorem 4.1). The function $x \rightarrow s_{x}(1, \ldots, 1)$ is harmonic with respect to the kernel $q^{-1} \stackrel{\circ}{P}(x, y)$. Let $q(\cdot, \cdot)$ be the kernel on $\mathscr{W}_{n}$ associated to $\widetilde{P}_{\mid Z(S U(n))}$. Then $q(x, y)=s_{y}(1, \ldots, 1) \stackrel{P}{P}(x, y) / s_{x}(1, \ldots, 1)$.

In other words under the embedding of $\stackrel{\circ}{W}_{n}$ into $L$ described in Section 4 , the Markov chain on $\stackrel{\circ}{W}_{n}$ is obtained from that on $L$ by conditioning the random walk on $L$, with respect to the dimension function, not to leave $\dot{W}_{n}$. The chain on $L$ itself is a nearest neighbor centered random walk.

Remark 4.1. In classical probability theory, the procedure of constructing the Markov kernel

$$
q(x, y)=p(x, y) h(y) / h(x)
$$

from a sub-Markov kernel $p$ and a nonnegative harmonic function $h$ is called Doob conditioning of $p$ with respect to $h$. Indeed, if $h$ is a minimal harmonic function, the Markov chain obtained after the conditioning process has probability one to tend in the Martin space $M S$ towards the element of the singleton of the support of the Martin measure $\mu_{h}$ of $h$ defined in Theorem 2.1.

Theorem 4.7 implies that the Martin boundary for these two Markov kernels is the same, and that multiplying by the function $x \rightarrow s_{x-\rho}(1)$ yields a one to one correspondence between harmonic functions for $\widetilde{P}$ on the center of $M(S U(n))$ and for $\stackrel{\circ}{P}$ on $\stackrel{\circ}{W}_{n}$. This theorem was a major motivation for introducing the operator $\stackrel{\circ}{P}$. In view of this, we can prove

Proposition 4.8. The minimal harmonic functions with respect to the operator $\stackrel{\circ}{P}$ (respectively $\widetilde{P}$ ) on $\stackrel{\circ}{W}_{n}$ are the functions

$$
x \rightarrow s_{x-\rho}\left(y_{1}^{\prime \prime}, \ldots, y_{n}^{\prime \prime}\right)
$$

(respectively $\left.x \rightarrow s_{x-\rho}\left(y_{1}^{\prime \prime}, \ldots, y_{n}^{\prime \prime}\right) / s_{x-\rho}(1)\right)$, where $\left(y_{1}^{\prime \prime}, \ldots, y_{n}^{\prime \prime}\right)$ run in $A_{q}$.

Proof. It is equivalent to have $f: \stackrel{\circ}{W} \rightarrow \mathbb{R}_{+}^{*}$ harmonic with respect to $\stackrel{\circ}{P}$, and $\tilde{f}: x \in \stackrel{\circ}{ }$. $f(x) / s_{x-\rho}(1)$ harmonic with respect to $\widetilde{P}$.

By [4], for any positive harmonic element $f$, there exists an unique finite positive measure $\mu_{f}$ on the set of positive elements of $S L(n)$ (we call it $S L(n)_{+}$), such that $f=\int_{S L(n)} i(A) d \mu_{f}(A)$. Since $f$ is invariant under $\mathrm{Ad}, \mu_{f}$ is also invariant under Ad. Let $\widetilde{\mu_{f}}$ be the image of $\mu_{f}$ under the canonical projection of $\operatorname{SL}(n)_{+}$onto $S L(n)_{+} /$Ad. The quotient space $S L(n)_{+} /$Ad contains naturally $A_{q}$, and the support of $\widetilde{\mu_{f}}$ is a subset of $A_{q}$ upon this inclusion. 
Conversely, a finite positive measure $d \mu$ in $A_{q}$ represents an element of $Z \widehat{(S U(n))}$. Indeed, let $Y$ be the matrix $\operatorname{diag}\left(y_{1}, \ldots, y_{n}\right)$. Recall that $i$ was defined at Eq. (3.4). Then,

$$
\int_{S U(n)} \int_{A_{q}} i\left(U Y U^{*}\right) d U d \mu
$$

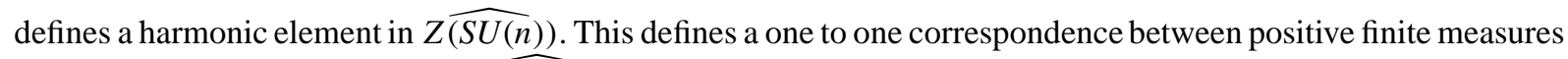
on $A_{q}$ and harmonic elements of $\left.Z \widehat{(S U(n)}\right)$.

Let $y=\left(y_{1} \geqslant \cdots \geqslant y_{n}\right) \in A_{q}$. Then for any $U \in S U(n)$ and for any $x \in \stackrel{\circ}{W}$, the trace of $U Y U^{*}$ in the irreducible representation corresponding to $x-\rho$ is $s_{x-\rho}\left(y_{i}\right)$. Therefore, denoting by $p_{x}$ the minimal central idempotent of $M(S U(n))$, the element $\int_{S U(n)} i\left(U M U^{*}\right) d U$ is harmonic, central and by equality of traces,

$$
p_{x} \int_{S U(n)} i\left(U M U^{*}\right) d U=p_{x} s_{x-\rho}(y) / s_{x-\rho}(1) .
$$

Therefore, to each element $y$ of $A_{q}$ corresponds the harmonic function

$$
x \rightarrow s_{x-\rho}(y) / s_{x-\rho}(1) .
$$

This proves $f$ has to be $x \rightarrow s_{x-\rho}(y) / s_{x-\rho}(1)$ for some $y \in A_{q}$.

Proof of second part of Theorem 4.1. $M B=M B_{\text {min }}$. According to Propositions 4.8 and 4.6, any point of $M B$ is indeed a minimal harmonic function. Furthermore, a minimal harmonic function corresponds to one and only one point of $\Sigma$ via the Martin kernel. Therefore the proof is complete.

\section{Quantum random walks on $\widehat{S U_{q}(n)}$ and Abelian subalgebras}

In this part, we apply the results of Section 4 to a quantum random walk on the dual of $S U_{q}(n)$ that was first introduced in [19].

\subsection{Quantum compact groups}

We start with the definition of matrix pseudogroup due to Woronowicz (see [33] and [32]). Let $A$ be a $C^{*}$-algebra with unit. The set $\mathbb{M}_{N}(A)$ of matrices with entries belonging to $A$ is identified with the $C^{*}$-algebra $B\left(\mathbb{C}^{N}\right) \otimes A$. A pair $\left(u=\left(u_{i j}\right) \in \mathbb{M}_{N}(A), A\right)$ is said to be a compact matrix pseudogroup iff

- the $*$-subalgebra $\mathcal{A}$ generated by matrix elements of $u$ is dense in $A$;

- there exists a $C^{*}$-homomorphism

$$
\Delta: A \rightarrow A \otimes A
$$

such that

$$
\Delta\left(u_{i j}\right)=\sum_{k=1}^{n} u_{i k} \otimes u_{k j}
$$

- $u$ is unitary and there exists a linear antimultiplicative mapping

$$
\kappa: \mathcal{A} \rightarrow \mathcal{A}
$$

such that $\kappa\left(\kappa\left(a^{*}\right)^{*}\right)=a$ and

$$
(\mathrm{id} \otimes \kappa) u=u^{-1} \text {. }
$$


An element $w=\left(w_{i j}\right) \in \mathbb{M}_{n}(\mathbb{C}) \otimes A$ is called a unitary corepresentation if the following holds:

$$
\Delta w_{i j}=\sum_{k} w_{i k} \otimes w_{k j}
$$

A vector space $=\mathcal{V}$ with basis $v_{i}$ and with a map $\Phi: \mathcal{V} \rightarrow \mathcal{V} \otimes A$ is called a comodule if there exists a corepresentation of $A$ such that

$$
\Phi v_{j}=\sum_{k} v_{k} \otimes w_{k j}
$$

For example, vect $\left\{w_{i 1}, \ldots, w_{i k}\right\}$ is a comodule if $w=\left(w_{i j}\right)$ is a unitary corepresentation. It is possible to define in an obvious way a notion of subcomodule, irreducible comodule, and equivalent comodules.

In this paper we shall focus on the specific example of $A\left(S U_{q}(n)\right)$. It is the universal $C^{*}$-algebra generated by $n^{2}$ elements $u_{k l}(k, l=1,2, \ldots, n)$ such that

$$
\begin{gathered}
\sum_{k} u_{k l}^{*} u_{k m}=\delta_{l m} I, \quad \sum_{k} u_{m k} u_{l k}^{*}=\delta_{l m} I, \\
\sum_{k_{1}, \ldots, k_{n}} u_{l_{1} k_{1}} \ldots u_{l_{n} k_{n}} E_{k_{1}, k_{2}, \ldots, k_{n}}=E_{l_{1}, l_{2}, \ldots, l_{n}} I
\end{gathered}
$$

where, for $q \in] 0,1]$,

$$
E_{i_{1}, i_{2}, \ldots, i_{n}}= \begin{cases}0 & \text { if } i_{k}=i_{l} \text { for some } k \neq l, \\ (-q)^{I\left(i_{1}, i_{2}, \ldots, i_{n}\right)} & \text { otherwise, }\end{cases}
$$

with $I\left(i_{1}, i_{2}, \ldots, i_{n}\right)$ denoting the number of inversed pairs in the sequence $\left(i_{1}, i_{2}, \ldots, i_{n}\right)$. Then $S U_{q}(n)=$ $\left(A\left(S U_{q}(n)\right), u\right)$ is a compact matrix pseudogroup. Furthermore, for $q=1$, it coincides with the algebra of continuous functions on the classical $S U(n)$ group.

For any matrix pseudogroup, there exists a unique state $h$ called Haar measure, satisfying

$$
h(x) \cdot 1=(h \otimes \mathrm{id}) \cdot \Delta(x)=(\mathrm{id} \otimes h) \cdot \Delta(x), \quad x \in A .
$$

The state $h$ is faithful in the case of $S U_{q}(n)$. Let $\left(\pi_{h}, H_{h}, \Omega_{h}\right)$ be the GNS triple of $h$, and $\Lambda_{h}$ the natural map from $A\left(S U_{q}(n)\right)$ to $B\left(H_{h}\right)$. The multiplicative unitary is defined as the bounded extension of the following operator:

$$
V\left(\Lambda_{h}(x) \otimes \xi\right)=\Delta(x)\left(\Omega_{h} \otimes \xi\right), x \in A, \quad \xi \in H_{h}
$$

$V$ is unitary and satisfies (see [2]) the following pentagon equation:

$$
V_{12} V_{13} V_{23}=V_{23} V_{12} \text {. }
$$

The dual von Neumann algebra $M\left(S U_{q}(n)\right)$ is the bicommutant in $B\left(H_{h}\right)$ of the set $\{($ id $\otimes \mu) V\}$ where $\mu$ runs over $B\left(H_{h}\right)_{*}$. The dual coproduct is defined by

$$
\widehat{\Delta}(x)=V^{*}(1 \otimes x) V
$$

therefore $M\left(S U_{q}(n)\right)$ is endowed with a Hopf-von Neumann algebra structure. This von Neuman algebra is well understood: since the representation theory of $S U_{q}(n)$ is the same as that of $S U(n)$, it has the same von Neumann algebra structure as the von Neumann algebra of $S U(n)$, therefore is isomorphic to

$$
\bigoplus_{x \in W} \mathbb{M}_{d_{x}}(\mathbb{C}) \text {. }
$$

For any representation $s \in W$, let $\left\{f_{z}\right\}_{z \in \mathbb{C}}$ be the family of Woronowicz characters. For its definition and basic properties, we refer to [32]. We only need to know that there exists a unique positive $\rho \in \mathbb{M}_{n}(\mathbb{C})$ with normalized 
trace, such that $f_{z}=\rho^{z}$, and $\rho$ intertwines the fundamental representation with its double contragradient. Let $s$ be the fundamental representation, $\mu=\operatorname{tr}_{s}(\rho \cdot)$, and

$$
\widetilde{P}:=(\mathrm{id} \otimes \mu) \widehat{\Delta} .
$$

The operator $\widetilde{P}$ is completely positive. It has already been considered by Izumi (see [16-18]). It leaves invariant the center $Z\left(S U_{q}(n)\right)$ of $M\left(S U_{q}(n)\right)$ (this is a consequence of [19], Lemma 3.2, (3)). In order to prove this, one needs to define the morphism $\Phi$ :

$$
M\left(S U_{q}(n)\right) \rightarrow M\left(S U_{q}(n)\right) \otimes L^{\infty}\left(S U_{q}(n)\right), \quad \Phi(x)=V(x \otimes 1) V^{*} .
$$

This is an action of $S U_{q}(n)$ on $M\left(S U_{q}(n)\right)$. The center $Z\left(S U_{q}(n)\right)$ is invariant under this action, therefore it makes sense to restrict it to Peter-Weyl blocks and to extend it to $\widehat{M\left(S U_{q}(n)\right)}$.

$\widetilde{P}$ intertwines $\Phi$, therefore it leaves invariant the center $Z\left(S U_{q}(n)\right)$ of $M\left(S U_{q}(n)\right)$. We define the twisted integer

$$
[n]_{q}=\frac{q^{n}-q^{-n}}{q-q^{-1}} .
$$

With this, one can show

Proposition 5.1. In the canonical basis of $\mathbb{M}_{n}(\mathbb{C})$, we have

$$
\rho=\frac{1}{[n]_{q}}\left(\begin{array}{cccc}
q^{-n+1} & 0 & \ldots & 0 \\
0 & q^{-n+3} & \ddots & \vdots \\
\vdots & \ddots & \ddots & 0 \\
0 & \ldots & 0 & q^{n-1}
\end{array}\right)
$$

It is a standard computation. See for example [32].

\subsection{Restrictions and h-processes}

Let $A\left(T^{n-1}\right)$ be the $C^{*}$-algebra of continuous function on the torus $T^{n-1}$. It is the universal Abelian $C^{*}$-algebra generated by the $n$ unitaries $u_{1}, \ldots, u_{n}$ satisfying $u_{1} \ldots u_{n}=1$. The coproduct $\widehat{\Delta} u_{i}=u_{i} \otimes u_{i}$ defines a $C^{*}$-Hopf algebra structure.

Let $\phi$ be the algebra morphism $A\left(S U_{q}(n)\right) \rightarrow A\left(T^{n-1}\right)$ such that $\phi\left(u_{i j}\right)=\delta_{i j} u_{i}$ with the induced relations. Obviously $\phi$ is a morphism of $C^{*}$-Hopf algebras. This allows to define a subalgebra $M\left(T^{n-1}\right)$ of $M\left(S U_{q}(n)\right)$. It is the von Neumann algebra generated by $\{(\mathrm{id} \otimes \mu) V\}$, where $\mu$ runs over the characters of $A\left(T^{n-1}\right)$. One can show that it is isomorphic to the group von Neumann algebra of $T^{n-1}$ and has a Hopf-von Neumann algebra structure. Therefore it makes sense to restrict $P$ defined at Eq. (5.5) to this von Neumann subalgebra. Furthermore, $\widetilde{P}$ is invariant under the adjoint action $\Phi$, thus it leaves invariant the center $Z\left(S U_{q}(n)\right)$.

Therefore, $\widetilde{P}$ restricts to two natural Abelian subalgebras of $M\left(S U_{q}(n)\right) . M\left(T^{n-1}\right)$ is in natural correspondence with $L^{\infty}(L)$ defined in Section 4 (the index function of $e_{i}$ corresponds to (id $\left.\otimes u_{i}\right) V$ in this identification). Similarly, the representation theory of $S U_{q}(n)$ and that of $S U(n)$ being the same for $q \in(0,1), Z\left(S U_{q}(n)\right)$ is naturally identified with $L^{\infty}\left(\stackrel{\circ}{W}_{n}\right)$. Thus $\widetilde{P}$ induces a classical Markov chain on $L$ and an other one on $\dot{W}_{n}$.

It is of natural interest to investigate a (probabilistic) link between these two Markov chains, for this has already been done by Biane in [6] in the case $q=1$ (see Theorem 4.7 and Remark 4.1).

Consider, for $q<1$, the point of $\Sigma \subset S^{n-2}$

$$
y_{q}=\sum_{i=1}^{n} q^{n+1-2 i} e_{i} /\left\|\sum_{i=1}^{n} q^{2 i-n-1} e_{i}\right\| \in S^{n-2} .
$$


Proposition 5.2. The random walk induced by the restriction of $\widetilde{P}$ to $M\left(T^{n-1}\right)$ corresponds on the lattice $L$, to the convolution by the probability measure

$$
\mu\left(e_{i}\right)=\frac{q^{-n+2 i-1}}{[n]_{q}} .
$$

It is obtained by conditioning, in Doob's sense of Remark 4.1, the sub-Markov random walk

$$
\tilde{\mu}\left(e_{i}\right)=\frac{1}{[n]_{q}}
$$

to converging towards the point $y_{q}$.

Proof. It is enough to remark that the function

$$
h:\left\{\begin{array}{l}
L \rightarrow \mathbb{R}, \\
\sum x_{i} e_{i} \rightarrow q^{\sum_{i} x_{i}(-n+2 i-1)}
\end{array}\right.
$$

is well defined and harmonic with respect to $\tilde{\mu}$. Furthermore the Doob conditioning of the convolution operator by $\tilde{\mu}$ with respect to $h$ is the convolution operator by $\mu$ and by the law of large numbers, this random walk almost surely tends to $y_{q}$.

Now we focus on the restriction to the center.

Proposition 5.3. The transition probability of the random walk restricted to the center is

$$
p_{l, l^{\prime}}= \begin{cases}\frac{s_{l^{\prime}}\left(q^{-n+1}, q^{-n+3}, \ldots, q^{n-1}\right)}{s_{l}\left(q^{-n+1}, q^{-n+3}, \ldots, q^{n-1}\right)[q]_{n}} & \text { if } \exists i, l^{\prime}=l+e_{i} \text { and } l^{\prime} \in \stackrel{\circ}{W}, \\ 0 & \text { otherwise. }\end{cases}
$$

Proof. This is a consequence of [19] and [15] together with the fact that the quantum dimension of the representation $l$ is

$$
s_{l}\left(q^{-n+1}, q^{-n+3}, \ldots, q^{n-1}\right) .
$$

This last fact results from the fact that the representation theory of $S U_{q}(n)$ and $S U(n)$ are the same, that the maximal torus remains non-deformed, and that we therefore have a Weyl character formula.

Theorem 5.4. The restriction of $\widetilde{P}$ to the center corresponds to the random walk on $L$ with increment $\alpha \sum \delta_{e_{i}} / n$ with $\alpha=n /[n]_{q}$, conditioned in Doobs's sense of Remark 4.1, to converging towards the point $y_{q}$, not to die, and not to hit $\partial W_{n}$.

Proof. According to the previous section, Proposition 4.8 and Theorem $4.1, s \rightarrow s_{x}\left(q^{-n+1}, q^{-n+3}, \ldots, q^{n-1}\right)$ is harmonic with respect to $\stackrel{\circ}{P}$ and in the Martin theory, it corresponds to the point $y_{q}$. This implies that the Doob conditioning of $\stackrel{\circ}{P}$ with respect to this harmonic function corresponds to conditioning to tend towards $y_{q}$ and remain inside $\stackrel{\circ}{P}$. But this operator is also $\tilde{P}$, as it was computed in Proposition 5.3.

\section{Acknowledgements}

This work was completed during the author's $\mathrm{PhD}$. The author would like to thank his advisor P. Biane for many useful conversations. He also thanks M. Izumi for explaining him and communicating early versions of [19], and R. Vergnioux for explanations about $S U_{q}(n)$.

Note added after submission: the author would like to thank the referee for a very careful reading of the manuscript and many useful remarks. 


\section{References}

[1] L. Accardi, A. Frigerio, J.T. Lewis, Quantum stochastic processes, Publ. Res. Inst. Math. Sci. 18 (1) (1982) $97-133$.

[2] S. Baaj, G. Skandalis, Unitaires multiplicatifs et dualité pour les produits croisés de $C^{*}$-algèbres, Ann. Sci. École Norm. Sup. (4) 26 (4) (1993) 425-488.

[3] P. Biane, R. Durrett, Lectures on probability theory, in: P. Bernard (Ed.), Lectures from the Twenty-third Saint-Flour Summer School held August 18-September 4, 1993, Springer-Verlag, Berlin, 1995.

[4] Ph. Biane, Équation de Choquet-Deny sur le dual d'un groupe compact, Probab. Theory Related Fields 94 (1) (1992) $39-51$.

[5] P. Biane, Marches de Bernoulli quantiques, in: Séminaire de Probabilités, XXIV, 1988/89, Springer, Berlin, 1990, pp. 329-344.

[6] P. Biane, Quantum random walk on the dual of su(n), Probab. Theory Related Fields 89 (1) (1991) 117-129.

[7] P. Biane, Some properties of quantum Bernoulli random walks, in: Quantum Probability \& Related Topics, World Scientific, River Edge, NJ, 1991, pp. 193-203.

[8] P. Biane, Minuscule weights and random walks on lattices, in: Quantum Probability \& Related Topics, World Scientific, River Edge, NJ, 1992, pp. 51-65.

[9] P. Biane, Théorème de Ney-Spitzer sur le dual de su(2), Trans. Amer. Math. Soc. 345 (1) (1994) $179-194$.

[10] G. Choquet, J. Deny, Sur l'équation de convolution $\mu=\mu * \sigma$, C. R. Acad. Sci. Paris 250 (1960) $799-801$.

[11] J. Dixmier, Les $C^{*}$-algèbres et leurs représentations, Gauthier-Villars \& Cie, Paris, 1964.

[12] E.G. Effros, Z.-J. Ruan, Discrete quantum groups. I. The Haar measure, Internat. J. Math. 5 (5) (1994) 681-723.

[13] W. Fulton, Young tableaux, Cambridge University Press, Cambridge, 1997. With applications to representation theory and geometry.

[14] W. Fulton, J. Harris, Representation Theory, Springer-Verlag, New York, 1991. A first course, Readings in Mathematics.

[15] F. Hiai, M. Izumi, Amenability and strong amenability for fusion algebras with applications to subfactor theory, Internat. J. Math. 9 (6) (1998) 669-722.

[16] M. Izumi, Actions of compact quantum groups on operator algebras, Sūrikaisekikenkyūsho Kōkyūroku 1024 (1998) 55-60. Profound development of operator algebras (Japanese) (Kyoto, 1997).

[17] M. Izumi, Actions of compact quantum groups on operator algebras, in: XIIth International Congress of Mathematical Physics (ICMP '97) (Brisbane), Internat. Press, Cambridge, MA, 1999, pp. 249-253.

[18] M. Izumi, Non-commutative Poisson boundaries and compact quantum group actions, Adv. Math. 169 (1) (2002) 1-57.

[19] M. Izumi, Non-commutative Poisson boundaries and compact quantum group actions, November 29, 2000.

[20] J.G. Kemeny, J.L. Snell, A.W. Knapp, Denumerable Markov Chains, second ed., in: Graduate Texts in Mathematics, vol. 40, SpringerVerlag, New York, 1976. With a chapter on Markov random fields, by D. Griffeath

[21] P.-A. Meyer, Éléments de probabilités quantiques. I-V, in: Séminaire de Probabilités, XX, 1984/85, Springer, Berlin, 1986, pp. 186-312.

[22] P.-A. Meyer, Éléments de probabilités quantiques. VI-VIII, in: Séminaire de Probabilités, XXI, Springer, Berlin, 1987, pp. 33-78.

[23] P.-A. Meyer, Eléments de probabilités quantiques. IX. Calculs antisymétriques et "supersymétriques" en probabilités, in: Séminaire de Probabilités, XXII, Springer, Berlin, 1988, pp. 101-123.

[24] P.-A. Meyer, Éléments de probabilités quantiques. X. Calculs avec des noyaux discrets, in: Séminaire de Probabilités, XXII, Springer, Berlin, 1988, pp. 124-128.

[25] P.-A. Meyer, Éléments de probabilités quantiques. X [bis]. Approximation de l'oscillateur harmonique (d'après L. Accardi et A. Bach), in: Séminaire de Probabilités, XXIII, Springer, Berlin, 1989, pp. 175-182.

[26] P.-A. Meyer, Éléments de probabilités quantiques. XI. Caractérisation des lois de Bernoulli quantiques d'après K.R. Parthasarathy, in: Séminaire de Probabilités, XXIII, Springer, Berlin, 1989, pp. 183-185.

[27] S. Neshveyev, L. Tuset, The Martin boundary of a discrete quantum group, 2002.

[28] P. Ney, F. Spitzer, The Martin boundary for random walk, Trans. Amer. Math. Soc. 121 (1966) 116-132.

[29] D. Revuz, Markov Chains, second ed., North-Holland, Amsterdam, 1984.

[30] M. Schürmann, M. Skeide, Infinitesimal generators on the quantum group $\operatorname{su}_{q}$ (2), Infin. Dimens. Anal. Quantum Probab. Relat. Top. 1 (4) (1998) 573-598

[31] D.V. Voiculescu, K.J. Dykema, A. Nica, Free Random Variables, American Mathematical Society, Providence, RI, 1992. A noncommutative probability approach to free products with applications to random matrices, operator algebras and harmonic analysis on free groups.

[32] S.L. Woronowicz, Compact matrix pseudogroups, Comm. Math. Phys. 111 (4) (1987) 613-665.

[33] S.L. Woronowicz, Tannaka-Krĕ̌n duality for compact matrix pseudogroups. Twisted su( $N)$ groups, Invent. Math. 93 (1) (1988) 35-76. 